\title{
An Experimental Investigation on Segregation Process of Binary Materials Based on Various Vibration Conditions
}

\author{
Bahaaulddin D. Mahdi, Nadhim M. Faleh* \\ Al-Mustansiriah University, Engineering College \\ Bab-Muadum, Baghdad, Iraq \\ * Corresponding author's email: dr.nadhim [AT] uomustansiriyah.edu.iq
}

\begin{abstract}
The mixing and segregation process of binary steel-glass mixture within a vertically vibrated containerat different frequencies are investigated in this paper. The image processing techniques areemployed to track the convection flow of granular materials. Six different amounts of frequency $(20,25,30,35,40$, and50 $\mathrm{Hz})$ for certain amplitudes are used to study the effects of miscellaneous vibration conditions on the velocity fields of moving beads.The results show that increasing frequency with constant amplitude has a positive effect on increasing the velocity movement of the beads in addition, the decreasing of amplitude at almost constant dimensionless vibrational acceleration cause the velocity movement of the beads to be increased.The granular bed was divided into three horizontal parts and a separate study was provided for each one.
\end{abstract}

Keywords--- Segregation process; Binary mixture; Velocity fields; Granular materials; Frequency

\section{INTRODUCTION}

There is a global use of granular materials in a wide range of industries e.g. chemical, food industries, pharmaceutical, powder metallurgy and mining (Chang et al. 2014, Zhou et al. 2016, Liu et al. 2018). In the industries there are many methods used to separate multiple materials, such as magnetic attraction, solubility, buoyancy, screening, chemical decomposition, etc. The understanding of segregation mechanisms for granular material is a key technological and scientific challenge to both scientists and engineers.

One of the well-studied segregation phenomena is the Brazil nut effect (Rosato et al. 1987, Knight et al. 1993, Cooke et al. 1996, Vanel et al. 1997, Breu et al. 2003, Srebro and Levine 2003, Kondic et al. 2003, Sanders et al. 2004, Ciamarra et al. 2006, Ulrich et al. 2007, Brito et al. 2008, Clement et al. 2010, Plantard et al. 2006, Metzger et al. 2011, Jain et al. 2013, Liao et al. 2014) which involves a large particle immersed in a container of smaller granular materials that vibrated by use of a shaker. This phenomenon provides the downward movement of the smaller particles under vibration. Shakers are one of the most important devices to mix and separate granular materials in modern industries (Suzuki et al. 1980, Pakowski et al. 1984, Yu 1992, Hunt et al. 1994). In the vibrated binary mixture, segregation may be resulted from differences in particle density, particle size, angle of repose and properties of the materials (Williams 1976, Johanson 1978, Hsiau and Yu 1997). Two convection cells could be found in the vibrated bed (Knight et al. 1996, Wassgren 1997, Hsiau and Chen 2000). Lee (1994) claimed the variation in density as reason for the convection motion. Granular material enclosed in tank and shacked vertically show the generation of convection rolls only with an acceleration amplitude above a threshold (1.2 g) (Hsiau et al. 2002). Mao-Bin et al. (2005) discussed the effect of container geometry on the segregation pattern. Of the several systems studied to investigate granular segregation, the vertically vibrated two-dimensional containers is a very useful geometry since it picks up most important details of granular flows and makes the controlling on all beads easier and simple to record.

Rosato et al. $(1986,1987,1991)$ explained the segregation phenomena occurred in a shaker by using a Monte Carlo algorithm simulation. Breu et al. (2003) found that the vibration conditions are an important factors affecting on particle segregation and should be well considered in the criterion. Recently, Sandali et al. (2017) studied segregation patterns in binary mixtures with same layer-thicknesses under vertical vibration. Cano-Pleite et al. (2017) performed segregation of equal-sized particles of different densities in a vertically vibrated fluidized bed. According to previous researches, segregation process of binary mixture at different frequencies has not been performed up to now. Literature review reveals that an inclusive understanding of the effects of external excitation on particle segregation is still lacking in research community.

The objective of this paper is to illustrate the behavior of binary mixture consisted of identical size steel and glass beads subjected to different vibration conditions. The granular bed was divided into three horizontal parts and a separate study was provided for each one. The effect of the frequency and amplitude on the velocity fields of granular bed are examined experimentally. Actually, six different amounts of frequency $(20,25,30,35,40$, and $50 \mathrm{~Hz}) \mathrm{for}$ certain amplitudes are used to study the effects of miscellaneous vibration conditions on the velocity fields of moving beads.Image processing techniqueis used to record and analyze the particle movement and convection flow mechanism. 
It will be indicated that increasing frequency with constant amplitude has a positive effect on increasing the velocity movement of the beads in addition, the decreasing of amplitude at almost constant vibrational acceleration cause the velocity movement of the beads to be increased.

\section{EXPERIMENTAL SETUP}

In Fig.1, the experimental apparatus has been illustrated. A vibration table having variable speed control is serving as the vertical shaker. The system is vertically driven by sinusoidal signals produced by a function generator. The vibrational frequency $f$ is adjusted by PID controller. Frequency range of system is $20 \mathrm{~Hz}-50 \mathrm{~Hz}$.
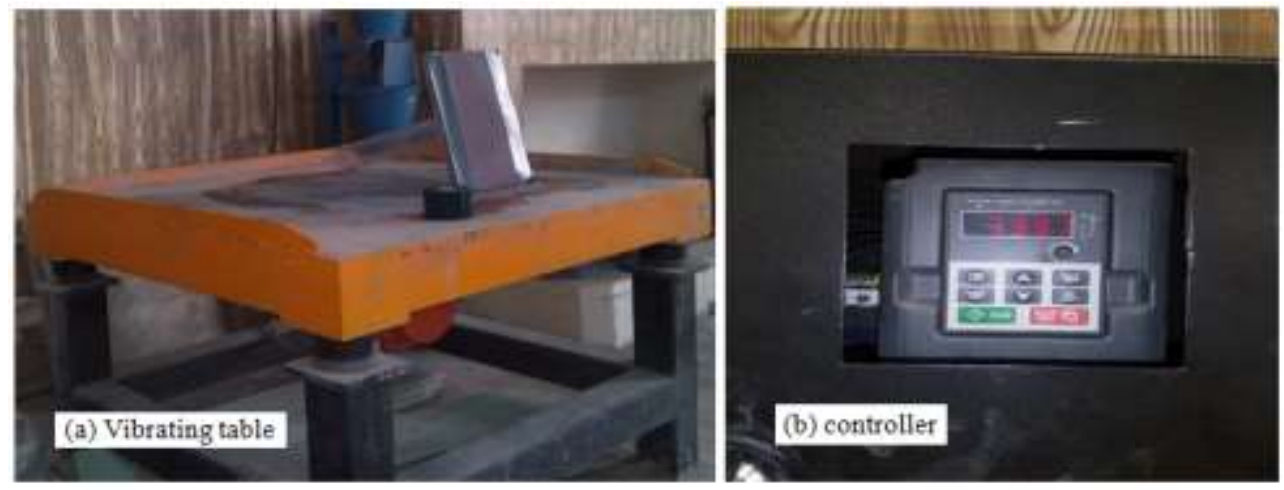

Fig 1.Experimental apparatus.

The vibrational radian frequency $\omega$ could be calculated from the relation $\omega=2 \pi f$ (Ebrahimi and Barati 2018, Yahia et al. 2015). Also, the amplitude $A$ can be defined as:

$A=\frac{a}{\omega^{2}}$

in which $a$ is vibrational acceleration. For further investigations, the non-dimensional form of vibration acceleration $\Gamma$ will be used:

$\Gamma=\frac{a}{g}$

where $g$ is the acceleration due to gravity.

A reservoir with glass plates as the front and back walls and with glossy tape as the side and bottom walls is driven by the shaker. The height, the width and the depth of the inside space of the tank were 280, 170 and $5 \mathrm{~mm}$, respectively. Therefore, there exists only one layer of particles in the tank and the flow generated in the granular bed was twodimensional ( $x$ : horizontal coordinate with origin in the center; $y$ : vertical coordinate with origin at the bed floor).

The Granular materials used in this experiment were glass beads with mean diameter $d_{g}$ of $4 \mathrm{~mm}$ and density $\rho_{g}$ of $2490 \mathrm{~kg} / \mathrm{m}^{3}$, and steel beads with mean diameter $d_{s}$ of $3.2 \mathrm{~mm}$ and density $\rho_{s}$ of $7822 \mathrm{~kg} / \mathrm{m}^{3}$. We record all particles movements in a thin glass tank in the six different variations of frequencies. The frequencies of vibration are as follows: $20 \mathrm{~Hz}, 25 \mathrm{~Hz}, 30 \mathrm{~Hz}, 35 \mathrm{~Hz}, 40 \mathrm{~Hz}$, and $50 \mathrm{~Hz}$ with different amplitudes and subsequently dimensionless acceleration. The direction of motion in vibrations is predominantly vertical, there is also a slight movement in the transverse direction. For each frequency, we recorded a short clip by a high speed (120 frames per second) camera. This FPS rate seems to be enough because by a simple mathematical calculation it is clear that even at our maximum vibration frequency $(50 \mathrm{~Hz})$ the camera can record at least maximum and minimum picks of our particles movements. After image acquisition, we have to prepare our images for the segmentation and object tracking. An image processing technique system was employed to digitally record and analyze the motions in the granular bed. The velocity and convection flow of every tracer particle could be calculated. In computer vision, image segmentation is the process of partitioning a digital image into multiple segments (sets of pixels, also known as super-pixels). The goal of segmentation is to simplify and/or change the representation of an image into something that is more meaningful and easier to analyze. Image segmentation is typically used to locate objects and boundaries (lines, curves, etc.) in images. More precisely, image segmentation is the process of assigning a label to every pixel in an image such that pixels with the same label share certain characteristics.

After segmentation, we need to track all the beads and obtain all routes that every single bead passes. There are many tracking methods that are based on different features of movement for tracking desired objects. For example, optical flow 
based trackers track objects with the most flow of motions or template matching trackers finds pixels with the most likeness to the pixels from objects in the previous frames.

During this study, the container was divided into three parts: upper, middle, lower field as shown in Fig 2. The movement of particles in each part will be studied and analyzed separately. The reason for this is to find out the effect of particles high from the container base on mixing and segregation.

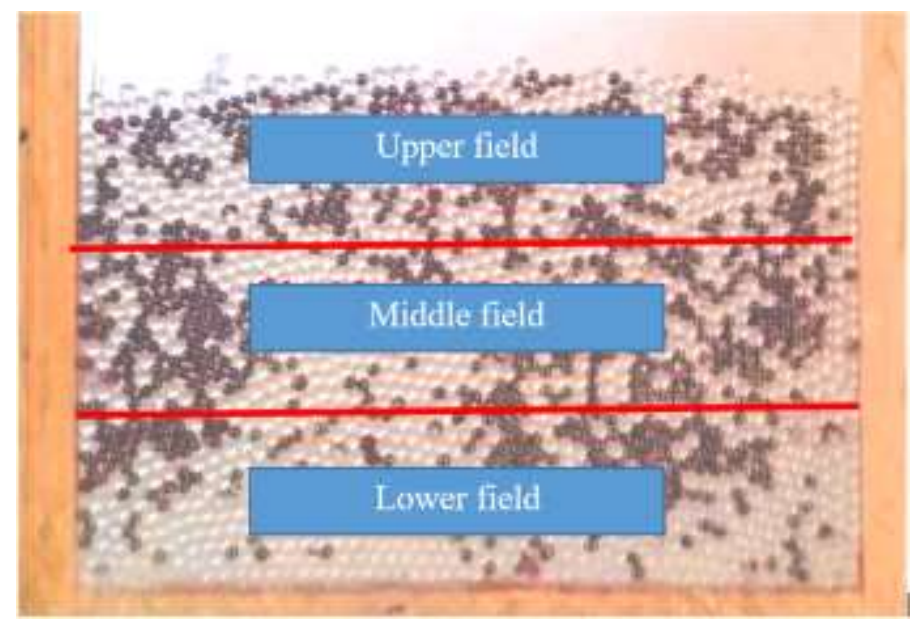

Fig 2.The upper, middle, and lower parts of vibration bed.

\section{RESULTS AND DISCUSSIONS}

In this section, obtained results for the segregation process of the binary steel-glass material will be presented based on different frequency conditions. The effects of frequency magnitude/amplitude and vibration accelerationon velocity of tracked particles will be discussed.

In Fig.4, the flow diagrams of tracked particles at different frequency magnitude $(f)$ and frequency amplitude (A) are presented. Comparing the parts of this figure reveals many important factors in velocity movement of the beads. For example, parts (a) and (e) shows the doubling of the vibration frequency at constant amplitude and hence the quadruple of the vibration acceleration. It can be seen that at a fixed amplitude, increasing frequency alone has a positive effect on increasing the velocity movement of the beads and thus can play a useful role in segregation systems. Also, one can conclude from this figure that decreasing of amplitude at almost constant dimensionless vibrational acceleration $\Gamma$ causes the velocity movement of the beads to be increased, and thus increasing the amplitude of the vibrations can have a much more impressive effect on the velocity of the beads in their flow of movement. Also, parts (b), (c), and (d), represent the velocity field in almost constant dimensionless vibrational acceleration $\Gamma$ and a decreasing in the amplitude amount. It is showed that the amount of increase in velocity fields due to frequency increase is few if accompanied by a decrease of amplitude. This result obtained from comparing fig. 4 (a) and (e) hand with Fig.4 (b), (c), and (d) on the other hand.
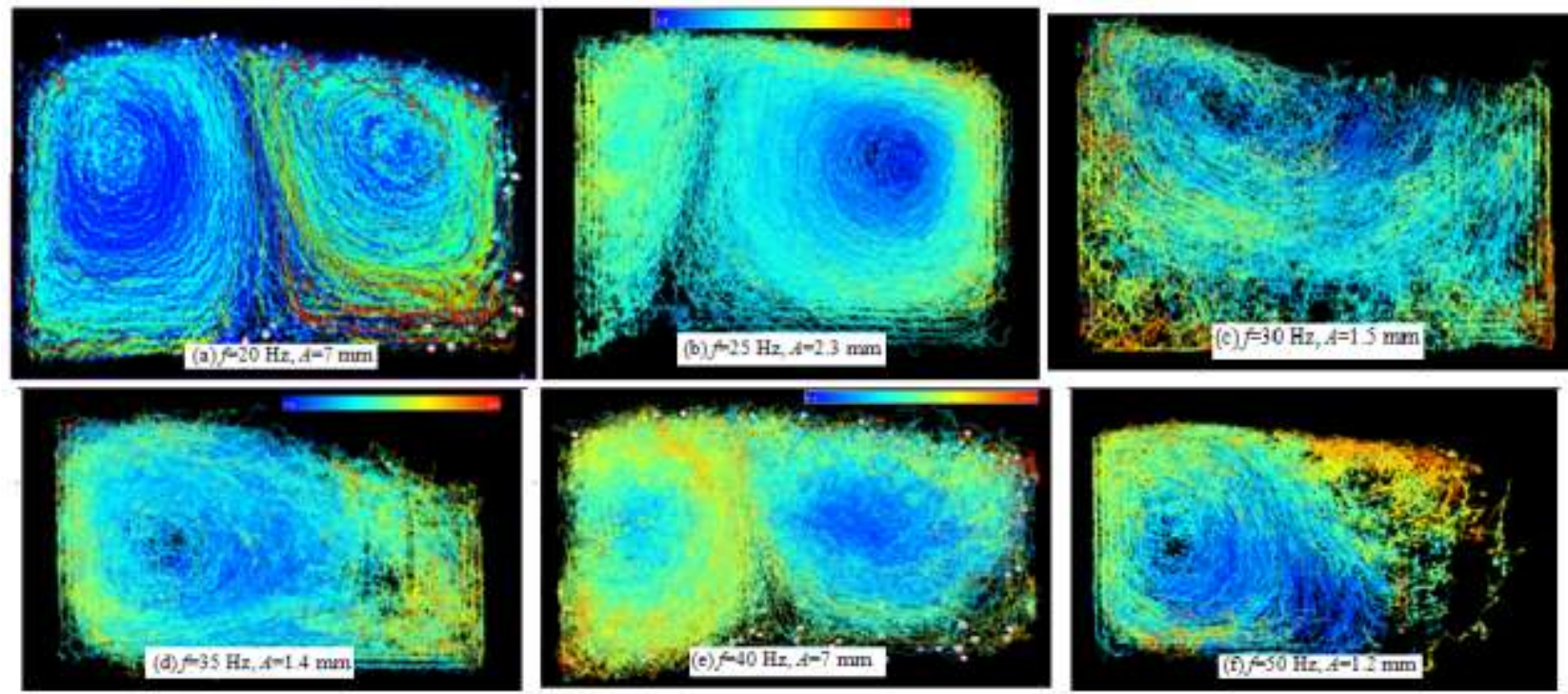

Fig 3. Flow diagram of tracked particles. 


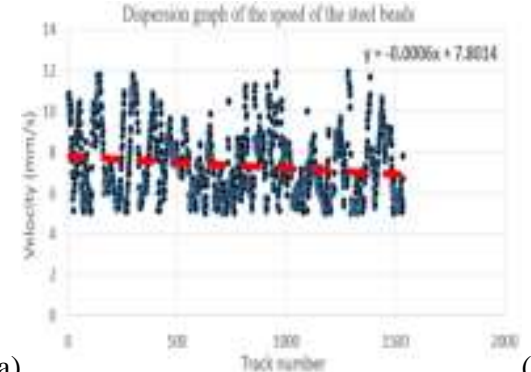

(a)

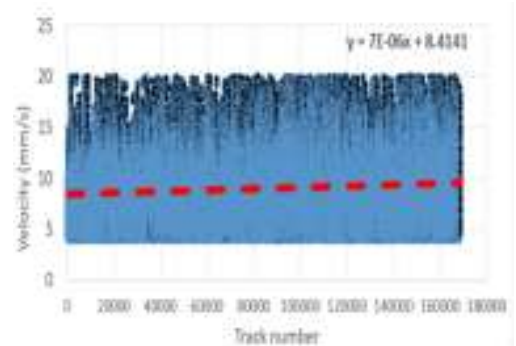

(b)

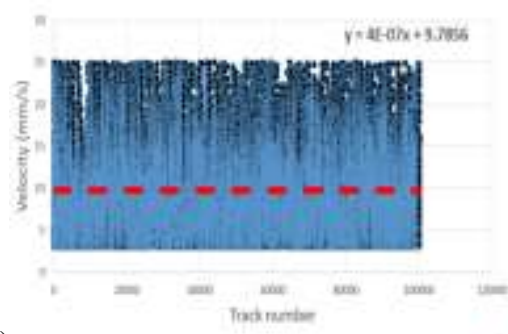

(c)

Fig 4. Velocity diagram of tracked particles for $f=20 \mathrm{~Hz}$ (a) the lower field, (b) the middle field, and (c) the upper field.

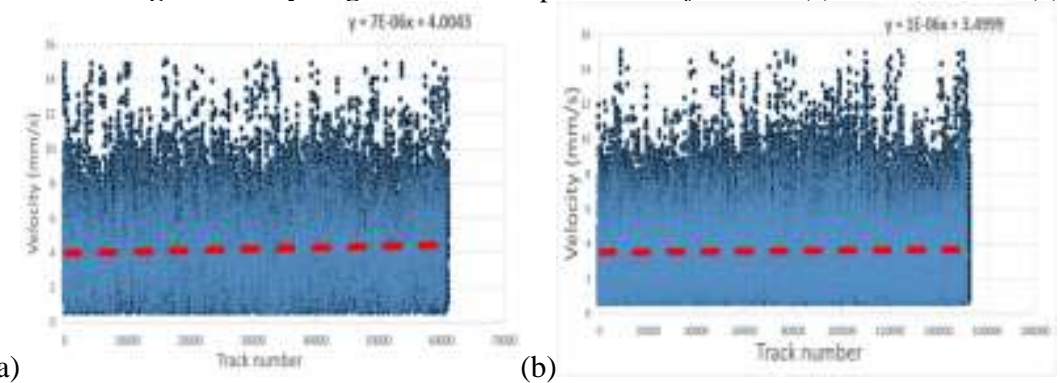

(c)

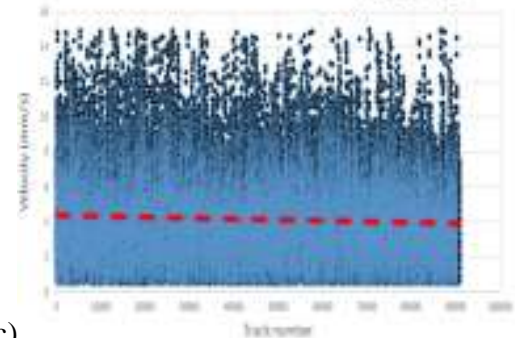

Fig 5. Velocity diagram of tracked particles for $f=25 \mathrm{~Hz}$ (a) the lower field, (b) the middle field, and (c) the upper field.
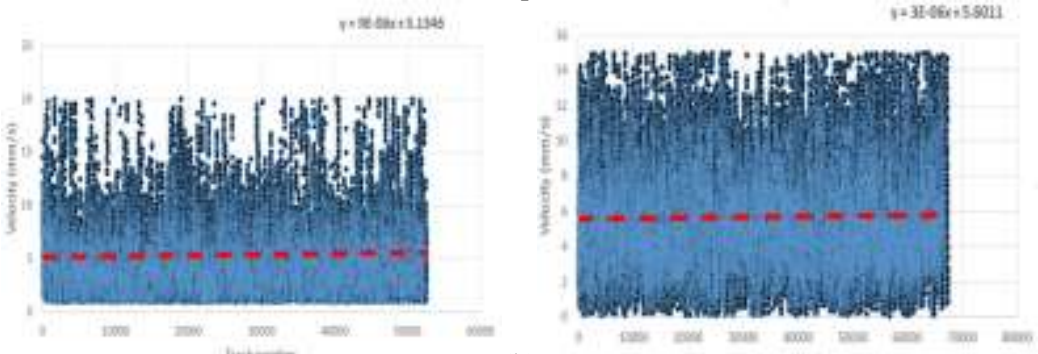

(b)

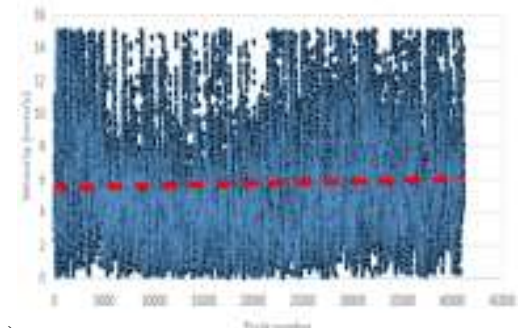

(c)

Fig 6. Velocity diagram of tracked particles for $f=30 \mathrm{~Hz}$ (a) the lower field, (b) the middle field, and (c) the upper field.
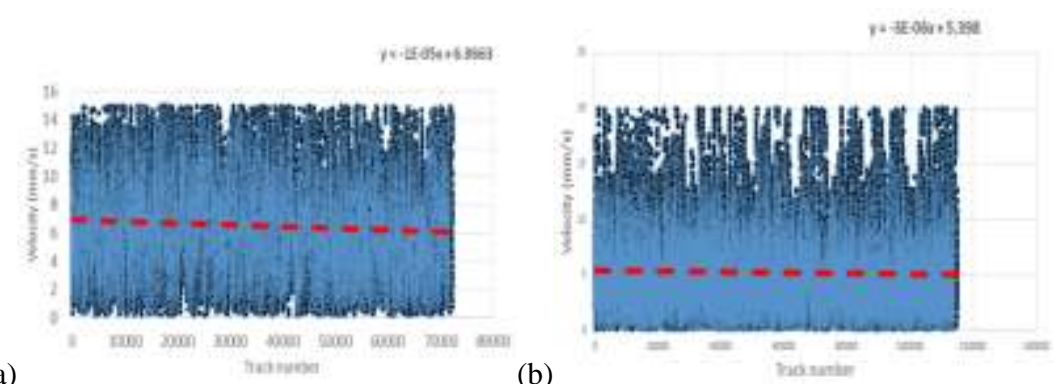

(b)

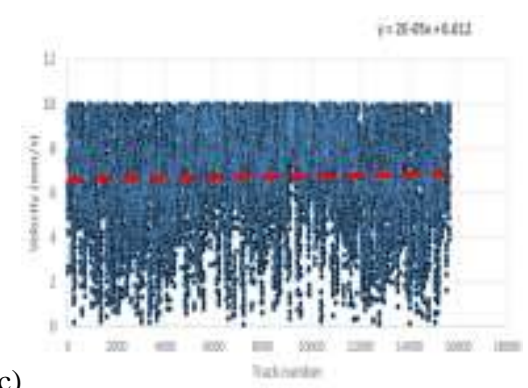

Fig 7. Velocity diagram of tracked particles for $f=35 \mathrm{~Hz}$ (a) the lower field, (b) the middle field, and (c) the upper field.

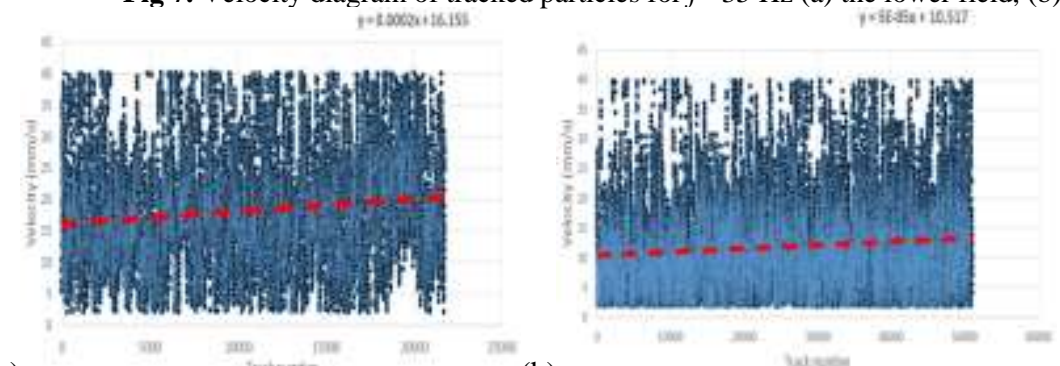

(a)

(b)

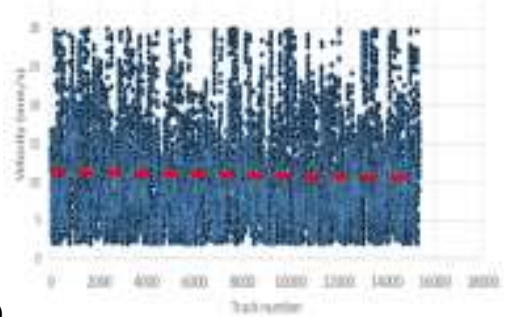

(c)

Fig 8. Velocity diagram of tracked particles for $f=40 \mathrm{~Hz}$ (a) the lower field, (b) the middle field, and (c) the upper field. 


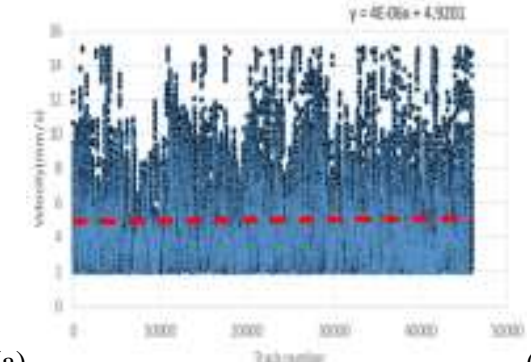

(a)

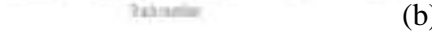

(b)

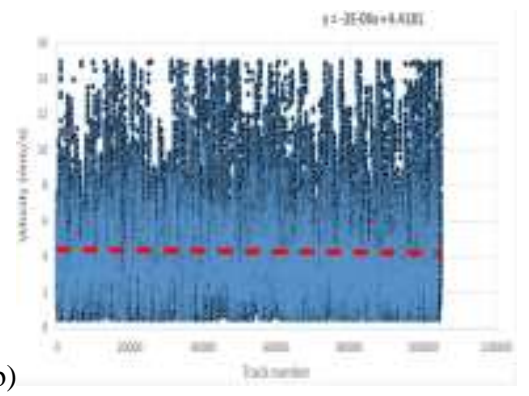

(c)

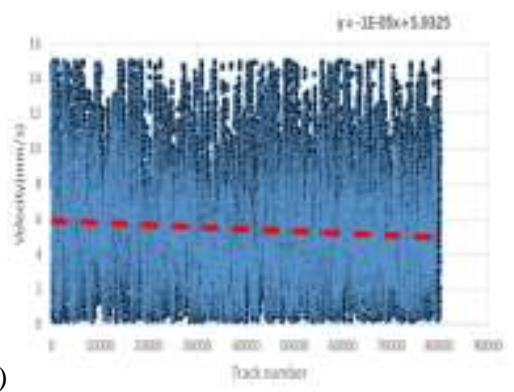

Fig 9. Velocity diagram of tracked particles for $f=50 \mathrm{~Hz}$ (a) the lower field, (b) the middle field, and (c) the upper field.

Illustrated in Figs. (4)-(9) is the velocity of particles with respect to track number at different values of vibration frequency $(f)$. Actually, the value of frequency is selected to be $f=20,25,40,35,40$ and $50 \mathrm{~Hz}$ in these figures. Each figure has three parts which are related to the lower, middle, and upper fields of the vibrating bed. The most important observation from these figures is that the number of moving particles in the middle part of the bed is higher than in the upper and lower parts at a prescribed frequency. Therefore, the middle part of the bed is the most important region due to the presence of highest moving particles. Hence, there are smaller numbers of moving particles at lower and upper fields of the bed. Also, the relationship between the particles velocity with the number of particles recorded in each region of the bed can be defined as an equation which is presented for each figure. In this equation, $y$ is particle velocity and $x$ is the number of tracked particles.Plotting this equation yields the dashed red line observable in the figures. One can see that the particle velocity can be controlled by changing of frequency $(f)$. For example, comparing the results of Figs. (7) and (8) which are related to frequencies $f=35 \mathrm{~Hz}$ and $40 \mathrm{~Hz}$ respectively reveals that the velocity of particles is decreasing with the increase of track number at $f=35 \mathrm{~Hz}$, but it is increasing with the increase of track number at $f=40 \mathrm{~Hz}$. However, at a prescribed track number, the velocity of particles at $f=35 \mathrm{~Hz}$ is smaller than that of $f=40 \mathrm{~Hz}$. As a general conclusion based on performed experiments with various frequencies, the highest particle velocity is obtained when the frequency is selected as $f=40 \mathrm{~Hz}$.

\section{CONCLUSION}

In this paper, experimental results were presented for the particle movement of two-dimensional vibrating granular bed in different vibration conditions. Spherical steel and glass beads of the same size were used to investigate the effect of the vertical vibrations conditions on their movement. The granular bed was divided into three horizontal parts and a separate study was provided for each one. The image processing technology were employed to measure the number of tracked particles and the velocity fields in each part. The results show that increasing frequency with constant amplitude has a positive effect on increasing the velocity movement of the beads in addition, the decreasing of amplitude at almost constant dimensionless vibrational acceleration cause the velocity movement of the beads to be increased. Also, the number of moving particles in the middle part of the vibrating bed is higher than in that of the upper and lower parts at a prescribed frequency.

\section{REFERENCES}

- Breu, A. P., Ensner, H. M., Kruelle, C. A andRehberg, I. (2003),"Reversing the Brazil-nut effect: competition between percolation and condensation. Physical review letters, 90(1), 014302.

- Brito, R., Enríquez, H., Godoy, S., \& Soto, R. (2008), “Segregation induced by inelasticity in a vibrofluidized granular mixture. Physical Review E, 77(6), 061301.

- Cano-Pleite, E., Hernández-Jiménez, F., Acosta-Iborra, A., Tsuji, T and Müller, C. R. (2017). "Segregation of equal-sized particles of different densities in a vertically vibrated fluidized bed. Powder Technology, 316, 101-110.

- Chang, J., Chu, X and Xu, Y. (2014). “A softening hyperelastic model and simulation of the failure of granular materials. Geomechanics and Engineering, 7(4), 335-353.

- Ciamarra, M. P., De Vizia, M. D., Fierro, A., Tarzia, M., Coniglio, A., \&Nicodemi, M. (2006). "Granular species segregation under vertical tapping: Effects of size, density, friction, and shaking amplitude. Physical review letters, 96(5), 058001 .

- Clement, C. P., Pacheco-Martinez, H. A., Swift, M. R., \& King, P. J. (2010). "The water-enhanced Brazil nut effect. EPL (Europhysics Letters), 91(5), 54001.

- Cooke, W., Warr, S., Huntley, J. M., \& Ball, R. C. (1996). "Particle size segregation in a two-dimensional bed undergoing vertical vibration. Physical Review E, 53(3), 2812.

- $\quad$ Ebrahimi, F., \& Barati, M. R. (2018). Wave propagation analysis of smart strain gradient piezo-magneto-elastic nonlocal beams. STRUCTURAL ENGINEERING AND MECHANICS, 66(2), 237-248. 
- Hsiau, S. S., \& Yu, H. Y. (1997). Segregation phenomena in a shaker. Powder Technology, 93(1), 83-88.

- Hsiau, S. S., \& Chen, C. H. (2000). Granular convection cells in a vertical shaker. Powder Technology, 111(3), 210-217.

- Hsiau, S. S., Wang, P. C., \& Tai, C. H. (2002). Convection cells and segregation in a vibrated granular bed. AIChE Journal, 48(7), 1430-1438.

- Hunt, M. L., Hsiau, S. S., \& Hong, K. T. (1994). Particle mixing and volumetric expansion in a vibrated granular bed. Journal of Fluids Engineering, 116(4), 785-791.

- Jain, A., Metzger, M. J., \&Glasser, B. J. (2013). Effect of particle size distribution on segregation in vibrated systems. Powder technology, 237, 543-553.

- Johanson, J. R. (1978). Particle segregation... and what to do about it. Chem. Eng., 85, 183-188.

- $\quad$ Kondic, L., Hartley, R. R., Tennakoon, S. G. K., Painter, B., \&Behringer, R. P. (2003). Segregation by friction. EPL (Europhysics Letters), 61(6), 742

- Knight, J. B., Jaeger, H. M., \& Nagel, S. R. (1993). Vibration-induced size separation in granular media: The convection connection. Physical review letters, 70(24), 3728.

- Knight, J. B., Ehrichs, E. E., Kuperman, V. Y., Flint, J. K., Jaeger, H. M., \& Nagel, S. R. (1996). Experimental study of granular convection. Physical Review E, 54(5), 5726.

- Lee, J. (1994). Heap formation in two-dimensional granular media. Journal of Physics A: Mathematical and General, 27(9), L257.

- $\quad$ Liao, C. C., Hsiau, S. S., \& Wu, C. S. (2014). Combined effects of internal friction and bed height on the Brazil-nut problem in a shaker. Powder Technology, 253, 561-567.

- $\quad$ Liu, S., Mao, H., Wang, Y., \&Weng, L. (2018). Experimental study on crushable coarse granular materials during monotonic simple shear tests. GEOMECHANICS AND ENGINEERING, 15(1), 687-694.

- Mao-Bin, H., Xiang-Zhao, K., Qing-Song, W., \& Yong-Hong, W. (2005). Effects of container geometry on granular segregation pattern. Chinese Physics, 14(9), 1844.

- Metzger, M. J., Remy, B., \&Glasser, B. J. (2011). All the Brazil nuts are not on top: Vibration induced granular size segregation of binary, ternary and multi-sized mixtures. Powder technology, 205(1-3), 42-51.

- $\quad$ Pakowski, Z. (1984). Theory and application of vibrated beds and vibrated fluid beds for drying processes. Advances in Drying, 3, 85-99.

- Plantard, G., Saadaoui, H., Snabre, P andPouligny, B. (2006). Surface-roughness-driven segregation in a granular slurry under shear. EPL (Europhysics Letters), 75(2), 335.

- Rosato, A.,Strandburg, K. J., Prinz, F andSwendsen, R. H. (1987). Why the Brazil nuts are on top: Size segregation of particulate matter by shaking. Physical Review Letters, 58(10), 1038.

- $\quad$ Rosato, A., Prinz, F., Standburg, K. J., \&Swendsen, R. (1986). Monte Carlo simulation of particulate matter segregation. Powder Technology, 49(1), 59-69.

- Rosato, A. D., Lan, Y., \& Wang, D. T. (1991). Vibratory particle size sorting in multi-component systems. Powder Technology, 66(2), 149-160.

- Sandali, Y., Xu, C., Zheng, N., Sun, G., \& Shi, Q. (2017). Segregation patterns in binary mixtures with same layerthicknesses under vertical vibration. arXiv preprint arXiv:1701.06850.

- Sanders, D. A., Swift, M. R., Bowley, R. M., \& King, P. J. (2004). Are Brazil nuts attractive?. Physical review letters, 93(20), 208002.

- Srebro, Y., \& Levine, D. (2003). Role of friction in compaction and segregation of granular materials. Physical Review E, 68(6), 061301.

- $\quad$ Suzuki, K., HOSAKA, H., YAMAZAKI, R., \& JIMBO, G. (1980). Drying characteristics of particles in a constant drying rate period in vibro-fluidized bed. Journal of Chemical Engineering of Japan, 13(2), 117-122.

- Ulrich, S., Schröter, M., \&Swinney, H. L. (2007). Influence of friction on granular segregation. Physical Review E, 76(4), 042301 .

- Vanel, L., Rosato, A. D., \& Dave, R. N. (1997). Rise-time regimes of a large sphere in vibrated bulk solids. Physical review letters, 78(7), 1255. 
- Wassgren, C. R. (1997). Vibration of granular materials(Doctoral dissertation, California Institute of Technology).

- Williams, J. C. (1976). The segregation of particulate materials. A review. Powder technology, 15(2), 245-251.

- Yahia, S. A., Atmane, H. A., Houari, M. S. A., \&Tounsi, A. (2015). Wave propagation in functionally graded plates with porosities using various higher-order shear deformation plate theories. Structural Engineering and Mechanics, 53(6), 11431165 .

- Yu, S. H. (1992). Drying performance and heat transfer in a vibrated fluidized beds. Drying'92, A., 731-740.

- Zhou, L., Chu, X., Zhang, X and Xu, Y. (2016), "Numerical investigations on breakage behaviour of granular materials under triaxial stresses. GEOMECHANICS AND ENGINEERING, 11(5), 639-655. 